\title{
Controversies of chemotherapy for the treatment of metastatic breast cancer
}

\author{
Pier Franco Conte*, Carmelo Bengala, Valentina Guarneri \\ Division of Medical Oncology, Department of Oncology and Haematology, University of Modena and Reggio Emilia, Modena, Italy
}

\section{ARTICLE INFO}

\section{Keywords:}

Metastatic breast cancer

Anthracycline

Taxane

Antimetabolite

HER2

Adjuvant failure

Oligometastatic disease

\begin{abstract}
Metastatic breast cancer (MBC) remains an incurable disease. The aims of treatment include tumour shrinkage, symptom control, delay of disease progression and prolongation of survival while maintaining an acceptable quality of life. In the last decade, a decline in mortality has been observed. Combination chemotherapy generally provides some survival advantage over single-agent chemotherapy. Taxanes and antimetabolites are among the most effective agents, providing a balance between efficacy and tolerability. Increasing numbers of patients are receiving adjuvant anthracycline and taxane therapy. In these patients, treatment options include cytotoxic agents not used in adjuvant treatment, re-challenge with anthracycline and taxanes, or new targeted agents such as pertuzumab, lapatinib or bevacizumab. Biology of the disease at cell level plays a major role in treatment choice. Key points in the intracellular signal transduction pathways relevant for cell proliferation, apoptosis and the angiogenesis/metastasis process, represent possible targets for new target-specific agents. Tailored therapies are a step forward in improving patients' prognosis.
\end{abstract}

(c) 2007 Elsevier Ltd. All rights reserved.

\section{Introduction}

Breast cancer is the most commonly diagnosed cancer in women worldwide with 1.15 million cases diagnosed according to Globocan 2002 data, and is the most prevalent cancer in women, with 4.4 million surviving up to 5 years following diagnosis ${ }^{1}$. Data show that approximately $50 \%$ of new cases and breast cancer deaths occur in developing countries, underlining the fact that the disease is a global concern ${ }^{1}$. Fortunately, in most countries a decline in mortality has been observed despite the continuous increase in diagnosis of new

${ }^{*}$ Corresponding author. Pier Franco Conte, MD, Professor of Oncology, Chief, Division of Medical Oncology, Director, Department of Oncology and Haematology, University Hospital, via del Pozzo 71, 41100 Modena, Italy. Tel: +39059 4224538; fax: +390594224429.

E-mail address: conte.pierfranco@unimo.it (P.F. Conte). cases. In fact, the increased incidence demonstrates that primary prevention of breast cancer, at least for the moment, is not feasible, while the increased efficacy of secondary prevention (screening) and more active treatments have led to both earlier detection and an improved cure rate. The majority of breast cancer patients are cured in the adjuvant setting. However, even in the case of metastatic breast cancer (MBC), survival prolongation has been achieved, with a fraction of patients surviving beyond 5 years.

\section{MBC: Disease characteristics and desirable treatment endpoints}

In spite of a screening programme, early diagnosis and the efficacy of adjuvant post-operative therapies, a significant proportion of breast cancer patients still 
develop distant metastases. Until recently, the proportion of patients in Italy who developed metastatic disease was 25-30\%; however, according to the most recent data from the Modena Cancer Registry, this proportion has now fallen to less than $20 \%{ }^{2}$. Thus, in developed countries, probably more than $80 \%$ of all breast cancer patients are effectively cured and will never develop metastases. According to the database of University Hospital, Modena, Italy, metastatic spread is diagnosed at a mean age of 66 years, by which time a significant proportion of patients already suffer with co-morbidities. The majority of patients $(70-80 \%)$ who develop metastases have failed adjuvant chemotherapy, usually on cyclophosphamide, methotrexate and 5-fluorouracil (CMF), but more recently, patients have increasingly been treated with anthracycline and taxanes. The median recurrencefree survival time from primary tumour diagnosis to metastatic spread is approximately 3 years. Most metastatic patients have visceral metastases (50-60\%) and/or bone metastases (65-75\%), and approximately $20 \%$ have human epidermal growth factor receptor-2 (HER2)-overexpressed disease.

The desirable treatment endpoints for patients with $\mathrm{MBC}$ are prolongation of survival, achieving symptomatic control, tumour shrinkage and maintaining or improving quality of life (QoL). Unfortunately, cure is unattainable for the vast majority of these patients. Therefore, in order to maintain an optimal QoL, it is important to balance the efficacy of treatment with its toxicity.

Traditionally, hormone receptor status has been the main characteristic used in clinical practice for deciding how to best treat MBC. Tumours with a positive oestrogen receptor (ER) or progesterone receptor (PR) status are usually treated with endocrine therapy, whereas those patients with a negative hormone receptor status will only respond to chemotherapy or trastuzumab. Other important characteristics include the diseasefree interval (DFI), the rate of tumour growth (slowgrowing tumours are more often treated with endocrine therapy, whereas more aggressive tumours are treated using chemotherapy), the site and extent of metastatic spread in the viscerae, and the performance status of the patient. Finally, all patients, even those who have received several lines of endocrine therapy, will eventually require chemotherapy as the tumour becomes hormone refractory.

\section{Management of MBC}

In attempting to establish which type of chemotherapy provides maximum benefit for endocrine-resistant patients with $\mathrm{MBC}$, it is useful to review the data available from different periods of chemotherapy development.

\subsection{The anthracycline era}

The survival of patients with MBC has improved over recent decades. This is due to several factors including stage migration and more favourable biological parameters. However, the availability of new active agents is a key factor in explaining the improved outcome ${ }^{3,4}$.

During the anthracycline era, quality of response to first-line chemotherapy was clearly the best predictor of long-term outcome. In patients receiving firstline treatment with 5-fluorouracil, doxorubicin and cyclophosphamide (FAC) or similar combinations, only those patients who achieved a complete clinical response (CR) had a more prolonged survival, while those patients who achieved a partial response, stabilisation of disease or no response attained a poorer and shorter survival irrespective of any salvage treatment ${ }^{5}$.

\subsection{The taxane era}

More recently, taxanes have been developed as very active agents for treating breast cancer. However, randomised clinical trials have failed to consistently prove the superiority of first-line regimens that included this novel class of agents. The activity of taxanes as salvage treatment, the difficulty in developing anthracycline plus taxane-based combinations and the limited statistical power of many trials may explain the disappointing results obtained. However, a meta-analysis recently published by the Cochrane Collaboration clearly indicated that, in general, taxane-containing regimens improved overall survival, time to progression and overall response versus non-taxane regimens in patients with $\mathrm{MBC}^{6}$.

\section{Quality of response as a surrogate endpoint for survival}

Quality of response to first-line chemotherapy was clearly a valid predictor of long-term outcome when very few effective salvage agents were available. As objective responses and, in particular, complete responses were more frequently observed with combination regimens, combination chemotherapy was commonly considered superior to single-agent regimens. This assumption was challenged when effective salvage cytotoxic agents became available. In fact, as discussed above, some trials failed to show clear superiority of combination regimens over sequential administration of single agents ${ }^{7,8}$. Again, however, two meta-analyses that included data from randomised clinical trials ${ }^{9}$ and from a single institution database ${ }^{10}$ confirmed that combination chemotherapy is better than single-agent treatment and that response to first-line chemotherapy is the best predictor of survival irrespective of second-, third- or fourth-line treatment. 
Table 1 - Treatment options according to prior adjuvant therapy in the 'old' scenario

\begin{tabular}{lllll} 
Treatment & \multicolumn{3}{c}{ Prior adjuvant treatment } \\
\cline { 2 - 5 } & \multicolumn{2}{c}{ No anthracycline } & Anthracycline & Anthracycline + taxanes \\
\hline 1st-line & Anthracycline-based regimens & Anthracycline + taxanes & Taxanes \pm antimetabolite 1 & Antimetabolite 1 \\
2nd-line & Taxanes \pm antimetabolite 1 & Antimetabolite 1 & Antimetabolite 2 & Antimetabolite 2 \\
3rd-line & Antimetabolite 2 & Antimetabolite 2 & & \\
\hline
\end{tabular}

Other commonly used drugs: vinorelbine, liposomal doxorubicin, or cyclophosphamide, methotrexate and 5 -fluorouracil. Re-challenge can be tested when treatment-free interval $>1$ year.

\section{The 'old' treatment scenario}

All these data, however, must be interpreted with caution as most patients who participated in the trials studied in these meta-analyses belonged to the 'old' scenario where adjuvant treatments rarely included anthracyclines. Thus, in case of relapse, several therapeutic options were still available, from anthracycline-based regimens to taxanes and antimetabolites (Table 1).

For example, after anthracycline failure a survival advantage could be attained by combining an antimetabolite with a taxane. Two randomised trials have shown the superiority of combination therapy. Docetaxel plus capecitabine was found to be superior to singleagent docetaxel, while gemcitabine plus paclitaxel was found to be superior to single-agent paclitaxel ${ }^{11,12}$. More recently, a randomised trial has shown that the combinations of gemcitabine plus docetaxel and capecitabine plus docetaxel are equally effective, although gemcitabine plus docetaxel is more tolerable ${ }^{13}$.

In summary, in the pre-anthracycline era, combination therapy - mainly CMF - proved more active than singleagent therapy; in the anthracycline era, anthracyclinecontaining combinations proved more effective than non-anthracycline-containing combinations. More recently, the availability of taxanes, either upfront or as a salvage treatment, has induced a further survival benefit. It should be noted that even in the taxane era, combination chemotherapy provided a limited but significant survival advantage over single-agent chemotherapy. A major advance, however, has been the development of trastuzumab, a monoclonal antibody against the HER2 receptor. Two randomised trials have shown that, in patients with HER2-overexpressing tumours, chemotherapy plus trastuzumab is significantly superior to chemotherapy alone in all treatment outcomes (response rate, progression-free survival and overall survival) ${ }^{14,15}$. Interestingly, in both trials, the combination of chemotherapy with trastuzumab produced better survival despite a significant proportion of patients randomised to chemotherapy alone having received trastuzumab upon progression. This observation is consistent with the preclinical data that demonstrated the synergistic interaction of several chemotherapeutic agents with trastuzumab in HER2-overexpressing cell lines ${ }^{16}$.
The patients included in all of these trials, however, belonged to the 'old' scenario. Some of these patients had never received adjuvant chemotherapy, CMF was still a common adjuvant regimen, only a small proportion of patients had failed on an adjuvant anthracycline, and even fewer had failed on adjuvant therapy with an anthracycline plus a taxane.

\section{The 'new' treatment scenario: biology drives choice}

Making treatment decisions in today's clinical practice is like trying to hit a moving target. Factors such as tumour biological profile, the site and extent of metastasis, the type of prior adjuvant treatments and, of course, patient characteristics and needs all require consideration. There is a need to discriminate between ER/PR-positive and -negative tumours, HER2-positive and -negative tumours, and to ascertain whether there is locoregional relapse or metastatic spread, where metastases are present, and whether they are visceral, bone or soft-tissue metastases.

In practice, patients with $\mathrm{MBC}$ are usually categorised according to low or moderate/high risk for death sooner rather than later. Patients at low risk have ER-positive cancer, HER2 overexpression (which predicts the efficacy of trastuzumab) and a long disease-free survival time (DFS) after primary surgery, with limited metastatic spread in bone or soft tissue. High-risk patients, on the other hand, have ER-negative, HER2-negative cancer, a shorter DFS and extensive metastatic spread, with visceral metastases and involvement of vital organs. Thus, in today's 'new' scenario, tumour biology plays a major role in treatment choice. More patients are diagnosed with a limited tumour burden and the majority of patients have already failed on adjuvant anthracyclines or anthracycline plus taxane regimens. Are single-agent antimetabolites the only treatment option for this majority group of patients (Table 2)?

If this is indeed true, then it should be recognised that this situation is similar to that of patient treatment in the second- or third-line setting of the 'old' scenario: i.e., there are no data showing any survival advantage for a single agent in managing these patients. Therefore, the treatment of patients who have failed on adjuvant therapy with an anthracycline and a taxane represents an increasing and unmet clinical need. On the other 


\begin{tabular}{|c|c|c|c|c|}
\hline \multirow[t]{2}{*}{ Treatment } & \multicolumn{4}{|c|}{ Prior adjuvant treatment } \\
\hline & No anthrac & cline & Anthracycline & Anthracycline + taxanes \\
\hline 1st-line & Anthracycline-based regimens & Anthracycline + taxanes & Taxanes \pm antimetabolite 1 & Antimetabolite 1 \\
\hline 2nd-line & Taxanes \pm antimetabolite 1 & Antimetabolite 1 & Antimetabolite 2 & Antimetabolite 2 \\
\hline 3rd-line & Antimetabolite 2 & Antimetabolite 2 & & \\
\hline
\end{tabular}

Trastuzumab for IHC 3+ or FISH-positive patients. Other commonly used drugs: vinorelbine, liposomal doxorubicin, or cyclophosphamide, methotrexate and 5-fluorouracil. Re-challenge can be tested when treatment-free interval $>1$ year.

hand, we know that the interval between primary tumour and development of metastases (DFI) is an important prognostic and predictive parameter. Furthermore, several agents have shown activity after anthracyclines and taxanes. Options in this 'new' scenario include cytotoxic agents not used in the adjuvant setting such as capecitabine, vinorelbine, gemcitabine, vinflunine or ixabepilone, re-challenge with the same drugs already used as adjuvant treatment (anthracyclines, taxanes, aromatase inhibitors, trastuzumab), or new targeted agents such as pertuzumab, lapatinib or bevacizumab. Another important observation is that today more patients are diagnosed with limited tumour burden because of increasingly sophisticated diagnostic procedures such as magnetic resonance imaging and positron emission tomography. As a consequence, palliation is also no longer the main goal of treatment for metastatic disease as most patients have a good performance status and are asymptomatic.

\section{QoL predicts survival}

Treatment must be tailored to individual patient needs, from survival prolongation to symptomatic control and maintenance of QoL. Increasingly, there is a need to discuss the importance of QoL with patients when predicting survival in MBC. QoL scores after disease relapse that demonstrate a better mood, appetite and physical well-being are a significant prognostic indicator of improved survival ${ }^{17}$. This follows on from the observation by Greer et al. nearly 30 years ago that patients with a 'fighting spirit' or emphatic denial of the disease had a more positive outcome than those who stoically accepted the situation ${ }^{18}$.

There is also a requirement to manage the patient's needs and expectations, and to discuss treatment options with him or her after adjuvant anthracycline and taxane failure. With respect to treatment decisionmaking, patients mostly prefer to share control with their doctor rather than a relative ${ }^{19}$. This is important in that patient expectations have been shown to differ, often more positively, from those of healthcare professionals. For instance, a survey by Slevin ${ }^{19}$ of 100 patients with solid tumours indicated that they were willing to accept more aggressive therapies associated with a $1 \%$ chance of cure versus a $50 \%$ chance chosen by healthy controls, or the $10-15 \%$ chance of cure chosen by healthcare professionals. Cancer patients found a 12-month prolongation of life derived from aggressive treatment acceptable versus 24-60 months of prolongation in healthy control participants, and 12-24 months for healthcare professionals. Patients were also more willing to accept aggressive therapies than healthy controls or healthcare professionals with respect to symptomatic relief ${ }^{19}$. Thus, greater effort should be made to involve patients in decision-making and when treatment is being selected.

\section{Oligometastatic disease: A curable disease?}

For a small fraction of patients with MBC who have low-burden and chemosensitive disease there may be a possibility of cure. Low-burden disease is not easy to define but, in general, it involves several instances of locoregional relapse, and three or fewer sites of lung metastases, liver metastases or brain metastases. It is also associated with limited bone metastases.

A survey by Greenberg et al. demonstrated the prognostic relevance of tumour burden in patients with recurrent metastatic disease ${ }^{5}$. Data showed that the probability of achieving a CR to first-line chemotherapy was higher for patients who had a limited burden of chemotherapy. More importantly, the proportion of patients who maintained a CR after 5 years or more was directly dependent on the tumour burden at diagnosis of metastatic spread. To illustrate this, 464 of the 1581 patients in this survey had one metastatic site and of these $25 \%$ had a CR, whereas of the 288 patients with four or more metastatic sites only $8 \%$ achieved a CR. More than 1 in 5 of the patients who achieved a CR to first-line chemotherapy and who had a limited tumour burden at diagnosis still maintained a CR after 5 years ${ }^{5}$. Thus, for a small proportion of patients, longterm remission with standard chemotherapy regimens is a reality. However, the better prognosis of low burden $\mathrm{MBC}$ can be due to earlier diagnosis or simply to leadtime bias, and there may be no difference in OS between early- and late-diagnosed MBC. 
Oligometastatic disease, defined as low-burden disease, is amenable to local treatment capable of controlling detectable tumour sites. Several treatments have shown potential in curing oligometastatic disease ${ }^{20}$. These include surgery, radiofrequency ablation, gammaknife, vertebroplasty or orthopaedic surgery.

There are some long-term survivors following surgical resection of apparently solitary cerebral, hepatic and pulmonary metastases ${ }^{21-25}$. However, in most studies the patients had received systemic therapy after surgical resection. Prognostic factors associated with better prognosis after surgery include good performance status, long disease-free interval, complete resection of metastatic tumour, number of metastatic nodules $(<3)$ and size of lesions $(<2 \mathrm{~cm})$. Rivera et al. performed a retrospective analysis on 259 patients with stage IV breast cancer without evidence of disease (NED) after surgical resection of recurrent metastatic lesions. These patients were treated with chemotherapy and compared with a historical control group of 62 patients who had not received chemotherapy following surgery. The study showed an improvement in OS and DFS for patients with stage IV-NED treated with doxorubicin-based adjuvant chemotherapy ${ }^{26}$. This finding is in accordance with the belief that most of these patients had micrometastases at the time of their first recurrence. Newer techniques, such as a circulating tumour cell assay or reverse transcription-polymerase chain reaction assay to detect micrometastases early, might be able to distinguish those patients who would benefit from systemic therapy in addition to local therapy from those who would not benefit.

\section{Chemosensitive disease}

Patients with chemosensitive disease can be identified on the basis of their clinical characteristics and tumour biological profile. These are patients who have had no prior adjuvant chemotherapy, or a long DFI following adjuvant chemotherapy. Patients with HER2-positive tumours can be successfully treated with trastuzumab, while among triple-negative tumours there are highly chemoresistant as well as highly chemosensitive tumours. In future, the possibility may exist to identify specific markers of chemosensitivity, such as overexpression of TOPOII $\alpha$, low $\tau$ protein level and perhaps specific gene signatures ${ }^{27,28}$.

There are reports in the literature showing that a mutimodal approach, including chemotherapy and local therapies, may improve long-term survival in patients with limited visceral metastases. In these instances, prolonged median overall survival can be achieved leading to $35-80 \%$ and $22-50 \%$ of patients with lung and liver metastases, respectively, surviving beyond 5 years ${ }^{29-32}$.

\section{Conclusion}

At presentation, the majority of patients with $\mathrm{MBC}$ do not require palliation, and indeed this is not the goal of first-line treatment for most patients. The primary aim is prolongation of survival, which can be achieved using chemotherapy both as first-line treatment and following anthracycline failure. Inevitably, the need for palliation increases with disease progression. Taxanes and antimetabolites such as gemcitabine are among the most effective agents to be used in combination therapy, thereby providing an optimal balance between efficacy and tolerability. Combining certain chemotherapeutic and targeted agents can also achieve added benefit through synergistic efficacy. Finally, it must be remembered that cure may be a realistic goal of treatment for a small subset of patients with oligometastases and that biology plays a major role in treatment choice. Tailored therapies will likely represent a further step in improving the prognosis of these patients.

Financial disclosure

Professor Conte has acted as a consultant for Eli Lilly and Company.

\section{REFERENCES}

1. Parkin DM, Bray F, Ferlay J, Pisani P. Global Cancer Statistics 2002. CA Cancer J Clin 2005;55:74-08.

2. Federico M, Rashid I, Artioli ME, et al. I Tumori in provincia di Modena nel 2004. Registro Tumori della Provincia di Modena. ISBN: 88-89037-03-2. Available from: http://www.rtm.unimo.it.

3. Giordano SH, Buzdar AU, Smith TL, Kau SW, Yang Y, Hortobagyi GN. Is breast cancer survival improving? Cancer 2004;100:44-52.

4. Chia SKL, Speers C, Kang A, et al. The impact of new chemotherapeutic and hormonal agents on the survival of women with metastatic breast cancer (MBC) in a population based cohort. Proc Am Soc Clin Oncol 2003;22 [abstract 22].

5. Greenberg PA, Hortobagyi GN, Smith TL, Ziegler LD, Frye DK, Buzdar AU. Long-term follow-up of patients with complete remission following combination chemotherapy for metastatic breast cancer. J Clin Oncol 1996;14:2197-205.

6. Ghersi D, Wilcken N, Simes J, Donoghue E. Taxane containing regimens for metastatic breast cancer. Cochrane Database Syst Rev 2005, Issue 2, Art. No. CD003366, DOI:10.1002/14651858.

7. Sledge GW, Neuberg D, Bernardo P, et al. Phase III trial of doxorubicin, paclitaxel, and the combination of doxorubicin and paclitaxel as front-line chemotherapy for metastatic breast cancer: an intergroup trial (E1193). J Clin Oncol 2003;21:588-92.

8. Conte PF, Guarneri V, Bruzzi P, et al. Concomitant versus sequential administration of epirubicin and 
paclitaxel as first-line therapy in metastatic breast carcinoma: results for the Gruppo Oncologico Nord Ovest randomized trial. Cancer 2004:101:704-12.

9. Carrick S, Parker S, Wilcken N, Ghersi D, Marzo M, Simes J. Single agent versus combination chemotherapy for metastatic breast cancer. Cochrane Database Syst Rev 2005, Issue 2, Art. No. CD003372, DOI:10.1002/14651858.

10. Bruzzi P, Del Mastro L, Sormani MP, et al. Objective response to chemotherapy as a potential surrogate end point of survival in metastatic breast cancer patients. J Clin Oncol 2005;23:5117-25.

11. O'Shaughnessy J, Miles D, Vukelja S, et al. Superior survival with capecitabine plus docetaxel combination therapy in anthracycline-pretreated patients with advanced breast cancer: phase III trial results. J Clin Oncol 2002;20:2812-23.

12. Albain KS, Nag S, Calderillo-Ruiz G, et al. Global phase III study of gemcitabine plus paclitaxel (GT) vs. paclitaxel (T) as frontline therapy for metastatic breast cancer (MBC): first report of overall survival. Proc Am Soc Clin Oncol 2004;22 [abstract 510].

13. Chan S, Romieu G, Huober J, et al. Gemcitabine plus docetaxel (GD) versus capecitabine plus docetaxel (CD) for anthracycline-pretreated metastatic breast cancer (MBC) patients (pts): Results of a European phase III study. Proc Am Soc Clin Oncol 2005;23 [abstract 581].

14. Slamon DJ, Leyland-Jones B, Shak S, et al. Use of chemotherapy plus a monoclonal antibody against HER2 for metastatic breast cancer that overexpresses HER2. N Engl J Med 2001;344:783-92.

15. Marty M, Cognetti F, Maraninchi D, et al. Randomized phase II trial of the efficacy and safety of trastuzumab combined with docetaxel in patients with human epidermal growth factor receptor 2-positive metastatic breast cancer administered as first-line treatment: the M77001 study group. J Clin Oncol 2005;23:4265-74.

16. Pegram MD, Konecny GE, O'Callaghan C, Beryt M, Pietras R, Slamon DJ. Rational combinations of trastuzumab with chemotherapeutic drugs used in the treatment of breast cancer. J Natl Cancer Inst 2004;96:739-49.

17. Coates AS, Hurny C, Peterson HF, et al. Quality-of-life scores predict outcome in metastatic but not early breast cancer. International Breast Cancer Study Group. J Clin Oncol 2000;18:3768-74.

18. Greer S, Morris T, Pettingale KW. Psychological response to breast cancer: Effect on outcome. Lancet 1979;2:785-7.

19. Slevin ML, Stubbs L, Plant HJ, et al. Attitudes to chemotherapy: comparing views of patients with cancer with those of doctors, nurses, and general public. Br Med J 1990;301:46.

20. Tait CR, Waterworth A, Loncaster J, Horgan K, Dodwell $\mathrm{D}$. The oligometastatic state in breast cancer: hypothesis or reality. Breast 2005;14:87-93.
21. Friedel G, Linder A, Toomes $H$. The significance of prognostic factors for the resection of pulmonary metastases of breast cancer. Thorac Cardiovasc Surg 1994;42:71-5.

22. McDonald ML, Deschamps C, Ilstrup DM, Allen MS, Trastek VF, Pairolero PC. Pulmonary resection for metastatic breast cancer. Ann Thorac Surg 1994;58:15991602.

23. Pocard M, Pouillart P, Asselain B, Falcou MC, Salmon RJ. Hepatic resection for breast cancer metastases: results and prognosis (65 cases). Ann Chir 2001;126:413-20.

24. Selzner M, Morse MA, Vredenburgh JJ, Meyers WC, Clavien PA. Liver metastases from breast cancer: long term survival after curative resection. Surgery 2000;127:383-9.

25. Yashimoto M, Tada T, Saito M, Takahashi K, Uchida Y, Kasumi F. Surgical treatment of hepatic metastases from breast cancer. Breast Cancer Res Treat 2000;59:177-84.

26. Rivera E, Holmes FA, Buzdar AU, et al. Fluorouracil, doxorubicin and cyclophosphamide followed by tamoxifen as adjuvant treatment for patients with stage IV breast cancer with no evidence of disease. Breast J 2002;8:2-9.

27. Slamon D, Eiermann W, Robert N, et al. Phase III randomized trial comparing doxorubicin and cyclophosphamide followed by docetaxel $(\mathrm{AC} \rightarrow \mathrm{T})$ with doxorubicin and cyclophosphamide followed by docetaxel and trastuzumab (AC $\rightarrow \mathrm{TH})$ with docetaxel carboplatin and trastuzumab (TCH) in HER2 positive early breast cancer patients: BCIRG 006 study. Breast Cancer Res Treat 2005;94(Suppl 1):S5 [abstract 1].

28. Gown AM, Goldstein LC, Porter PL, et al. Multivariate analysis of expression of the microtubule-associated protein, tau, predicts improved progression free and overall survival in patients with metastatic HER-2negative breast cancers treated with docetaxel and vinorelbine plus filgrastim. Proc Am Soc Clin Oncol 2006;24 [abstract 543].

29. Bathe OF, Kaklamanos IG, Moffat FL, Boggs J, Franceschi D, Livingstone AS. Metastasectomy as a cytoreductive strategy for treatment of isolated pulmonary and hepatic metastases from breast cancer. Surg Oncol 1999;8:35-42.

30. Maksan SM, Lehnert T, Bastert G, Herfarth C. Curative liver resection for metastatic breast cancer. Eur J Surg Oncol 2000;26:209-212.

31. Murabito M, Salat A, Mueller MR. Complete resection of isolated lung metastasis from breast carcinoma results in a strong increase in survival. Minerva Chir 2000;55: 121-7.

32. Tait CR, Waterworth A, Loncaster J, Horgan K, Dodwell D. The oligometastatic state in breast cancer: hypothesis or reality. Breast 2005;14:87-93. 